\title{
Multiscale conformational dynamics probed by time-resolved circular dichroism from milliseconds to picoseconds
}

\author{
F. Hache and P. Changenet \\ Laboratoire d'optique et Biosciences, CNRS, INSERM, Ecole polytechnique, Institut \\ Polytechnique de Paris \\ 91128 Palaiseau cedex, France \\ francois.hache@polytechnique.edu
}

\begin{abstract}
Time-resolved circular dichroism has been developed for a few decades to investigate rapid conformational changes in (bio)molecules. In our group, we have come up with several experimental set-ups allowing us to study pico-nanosecond local phenomena in molecular systems as well as much slower effects occurring in proteins and DNA in the folding processes. After an overview of the worldwide realizations in this domain, we present emblematic experiments that we have carried out, spanning time domain from picoseconds to seconds.
\end{abstract}

\section{Introduction}

Conformation of (bio)molecules or molecular assemblies is known to play a paramount role in chemical reactivity and biological functions. Gaining information on their dynamics has always been sought to understand the fundamentals of many biological or chemical phenomena. Circular dichroism (CD) has been used for ages to get information on the 3D conformation of chiral compounds in solution. Indeed, the $\mathrm{CD}$ spectrum of biomolecules and chiral chemical species may often give a clear indication of their absolute configuration, either from established rules (excitonic coupling, octant rule, ...) or from empirical knowledge or even with the support of theoretical calculations such as molecular dynamics [1]. In a quite different domain, time-resolved spectroscopy has been implemented from the development of pulsed lasers and has proved to be an outstanding tool for probing the dynamics of light-triggered elementary reactions taking place in molecules. It has therefore been clear that combining these two optical techniques will give the opportunity to decipher the fundamental processes at stake in stereochemistry, be it a pure chemical process or connected to biological reactions. Implementation of this combination of techniques has however proved to be challenging due to their weak signals prone to artifacts [2] [3].

In this context, our group has been developing, for a few decades, complementary TRCD techniques in order to investigate either ultrarapid (i.e. in the subpico - nanosecond time domain) [4] or slower (i.e. in microsecond up to the second time domain) processes in organic and inorganic compounds and in biomolecules [5]. While local conformational changes which are restricted to a few atoms generally occur on the ultrarapid timescale, more global phenomena, such as the changes of protein or DNA secondary structure occur on much larger time scales.

In the next section, we will give a brief history of the development of ultrarapid TRCD experiments. Notably, we will retrace the various ideas put forward in this restrained community and discuss their achievements. We will then illustrate several applications of the TRCD detections that we have developed in our laboratory for measuring the conformational 
dynamics of chemical and biological samples, on a time scale spanning sub-picosecond up to a few seconds.

\section{A brief history of the development of ultrarapid TRCD}

$\mathrm{CD}$ is defined as the difference in the absorption of a chiral sample between left and rightcircularly polarized light. Measuring the temporal evolution of a $\mathrm{CD}$ signal on a short timescale, i.e. on the time scale shorter than nanoseconds, is of great interest for accessing the conformational changes of chiral compounds. The common principle of these experiments is based on measurements with a pump-probe geometry, an experimental technique widely used in the community of ultrafast spectroscopy. The principle is to use a short laser pulse (most often pico- or femtosecond one) to provoke a perturbation in the sample under investigation and then to measure the pump-induced CD changes in the sample with a second opticallydelayed laser pulse, named the probe. The time resolution of such optical experiments is only determined by the temporal overlap of the pump and the probe pulses. Although the principle of these experiments is rather simple, their implementation for TRCD detection is actually quite difficult. First of all, the TRCD signals are very weak, i.e. two to four orders of magnitude weaker than those of classical achiral transient absorption measurements, which corresponds to a sensitivity of a few tens of $\mu \mathrm{OD}$. Therefore, it is not so easy to extract them out of the noise level which is unavoidable with pulsed lasers. Second, the pump-probe geometry very often provokes polarization artifacts that can be very difficult to overcome. This is the reason why despite their recognized interest, experimental implementations of TRCD remain rather scarce.

Two main directions have been proposed for implementing a TRCD detection in a pump-probe set-up. The first one is to modulate the probe polarization between two circular or elliptical polarizations and then to monitor the difference in the probe intensity transmitted by the sample. This is the most straightforward technique, but it has the major disadvantage of measuring very small changes in the sample absorption over a very large achiral absorption background and, as discussed below, this technique can be very prone to artifacts [3]. The second possibility is to take advantage of the fact that linearly-polarized light becomes elliptically polarized when going through a chiral sample. Thus, several set-ups capable to measure pump-induced ellipticity changes have also been implemented, with the main advantage to eliminate the strong achiral background contribution to the TRCD measurements increasing their sensitivity.

The pioneers in the development of fast TRCD spectroscopy are Kliger and Simon who performed the first experiments with a nanosecond and a picosecond time resolution, respectively. In his seminal paper [6], Kliger implemented a TRCD detection on a nanosecond flash phololysis system. Generation of alternating elliptical polarizations of the probe synchronized with the pump laser was achieved thanks to a strained fused silica plate, allowing direct measurements of the pump-induced CD changes in the sample with a nanosecond time resolution. In 1989, Simon [7] extended the time resolution into the picosecond domain by implementing a TRCD detection on a picosecond pump-probe set-up. The modulation of the circular polarization of the probe was done with an electro-optic modulator and the detection of the weak intensity modulation due to the pump-induced CD changes in the sample was performed with a lock-in amplifier. Hache et al. implemented a similar set-up by using a Pockels cell to modulate the probe polarization generated from a femtosecond laser source to reach a sub-picosecond temporal resolution [2]. 
Although very straightforward, all those experiments are nevertheless very difficult to carry out, especially because of the existence of numerous alignment artifacts due to sample birefringence. Those problems were acknowledged very rapidly [2] [3] and a very stringent alignment procedure of the Pockels cell was proposed to overcome the problems [8]. Those artifacts however represented a very severe drawback in some experiments and in 2006, Niezborala et al. proposed a new technique for sub-picoscond TRCD measurements based on the pump-induced change of the probe ellipticity of a linearly-polarized beam passing through a chiral sample [9]. In this experiment, the probe ellipticity changes are measured with a Babinet-Soleil compensator providing a tunable phase retardation. By monitoring the change in probe ellipticity after the pump excitation, both the transient absorption or CD signals can be easyly accessed. Although this procedure is more time-consuming than the previous ones, it has the advantage of being free of most artifacts.

All these TRCD set-ups have in common to work at a definite probe wavelength. Therefore, accessing the global spectral response of the sample is very time-consuming and requires accumulation of measurements carried out for several probe wavelengths. The idea to use spectrally-broad femtosecond probe pulses has therefore motivated a lot of works. The main difficulty of these experiments relies in the fine control of the probe polarization which can be easily altered by the polarization and transport optics of the beam. The first broadband TRCD experiments were proposed independently by Trifonov [10] and Mangot [11] in 2010. In both cases, the broadband detection was achieved in the visible spectral domain with a white-light continuum. In the former case, the $\mathrm{CD}$ signals were directly measured by modulating the left and right circular polarization of the probe with a Pockel cell [10]. In the second case, the pump-induced ellipticity change of the probe polarization was monitored with a combination of a broadband quarter waveplate and two crossed polarizers [10]. Improvement of broadband TRCD detection was proposed in 2015 by the implementation of a heterodyned detection of the pump-induced ellipticity changes of the probe in visible spectral domain, leading to TRCD measurements with a precision of $0.01 \mathrm{mOD}$ i.e. $0.3 \mathrm{mdeg}$ [12]. Heterodyned detection which measures the amplitude and the phase changes of the probe beam gives a simultaneous access to the pump-induced $\mathrm{CD}$ and optical rotary dispersion (ORD) changes of the sample. It was also shown that the joint use of heterodyned dectection and singular value decomposition (SVD) could disentangle the contribution of the anisotropy to the TRCD signals. More recently, the first demonstration of the use of femtosecond broadband TRCD in the UV spectral domain was done by Opperman et al. in 2019 [13]. Using a subtle combination of 6 pulses and a polarization modulation with a photoelastic modulator, they were able to measure the TRCD spectra of Ru(bpy) ${ }_{3}{ }^{2+}$ in the $260-370 \mathrm{~nm}$ range with a sensitivity of $0.02 \mathrm{mOD}$, i.e. $0.6 \mathrm{mdeg}$.

It is noteworthy to mention the recent theoretical studies of Mukamel's and OlayaCastro's groups for isolating the chiral contribution to signals measured with two-dimensional optical spectroscopy configurations [14, 15]. First attempt to introduce chirality in twodimensional electronic spectroscopy was achieved by Engel et coll, in 2014 who studied the dynamics of electronic localization in the photosynthetic complexes [16]. Before ending this discussion on the technical aspects of ultrarapid TRCD, let us cite the impressive works done by Helbing's and Cho's groups towards the development of femtosecond VCD spectroscopy $[17,18]$. VCD that is concerned with IR absorption related to molecular vibrations is known to be extremely weak and it is a real challenge to obtain reliable TR-VCD signals. Nice experiments on transient $\mathrm{CD}$ of $\mathrm{C}-\mathrm{H}$ vibrations in $\mathrm{CoCl}_{2}$ and $\mathrm{NiCl}_{2}$ were published in 2008 [19]. 
Most of the works presented above addressed the technical aspects of TRCD spectroscopy, providing proof-of-principle devices with good sensitivity and time-resolution and/or a large frequency bandwidth. However, their comprehensive utilisation to investigate the reactivity of chiral systems in general is still scarce. Following his seminal work, Kliger's group studied a large variety of compounds, ranging from inorganic metal complexes to several proteins (myoglobin, hemoglobin, cytochrome c, phytochrome) or tRNA. A review can be found in ref. [20]. Similarly, Simon's group investigated the conformational dynamics of hemoproteins and bacterial photosynthetic reaction centers [21]. In the past decade, our group investigated sereval chemical and biological systems [4] such as binaphthol, carboxymyoglobin, Photoactive Yellow Protein and more recently the folding/unfolding dynamics of proteins [22]. Another interesting application of TRCD was carried out by Meyer-Ilse et al. who monitored the ring opening of 7-dehydrocholesterol in 1-2 psec upon UV photoexcitation. [23]. More recently, Scholtz et al. studied the relaxation of a polyfluorenephenylene copolymer after UV irradiation [24]. Finally, let us cite the work by Opperman et al.where using broadband TRCD, the authors could monitor the backbone dynamics in modified peptides with a picosecond resolution [25].

Before concluding this overview of TRCD, we would like to comment on the feasibility of using circular dichroism to obtain relevant pieces of information on conformational changes. Indeed, monitoring changes in $\mathrm{CD}$ at a given wavelength - difficult as it may be - is not informative enough by itself and must be complemented by other knowledge. A first indication may be the comparison of transient absorption and TRCD signals: if both are different, one can infer that the CD signal is the signature of a conformational change and not only some electronic artifact. But it is often important to rely on other piece of information that can be general rules (excitonic coupling, octant rule) or empirical knowledge about the CD spectra expected after irradiation. For further discussion, see [26]. In other cases, quantum chemical calculations can help interpreting the TRCD measurements [27, 28]. This issue is of primary importance for single-wavelength experiments, but will be less so when broadband measurements can be performed routinely. Indeed, comparing the full CD spectra before and after photoexcitation and using standard techniques such as SVD will be very informative in future experiments. In this respect, use of Synchrotron Radiation is very promising [29].

\section{Sub-picosecond-nanosecond timescale}

In this section, we describe two examples where TRCD gives information on the dynamics of conformation on ultrashort timescales. The first one deals with the change in the dihedral angle of Binaphtol derivatives upon photoexcitation and the second one with the conformational changes occurring in carboxy-myoglobin after the photodissociation of CO. Both experiments are based on the pump-probe principle, where a first ultrashort laser pule provokes a conformational change that is monitored by measuring the $\mathrm{CD}$ of a second pulse. By varying the optical delay between the two pulses, one can access the time evolution of the transient $\mathrm{CD}$ with a sub-picosecond resolution.

\section{1) Binaphtol derivatives}


The binaphtol (binol) is a molecule composed of two identical naphtol moieties connected by a $\mathrm{C}-\mathrm{C}$ bonds, the angle between them being the dihedral angle. This geometrical configuration is at the origin of the binol chirality which can exist as an $\mathrm{R}$ or an $\mathrm{S}$ enantiomer. The bisignate CD spectrum in the UV clearly stems from the excitonic coupling between the two naphthol moieties. Upon excitation of its $\pi-\pi^{*}$ transitions, the binol is known to undergo a change in the dihedral angle. TRCD measurements allow to follow in real time the dynamics of this geometrical change.

(a)

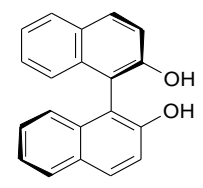

(R)-Binol

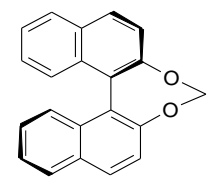

(R)-PL1

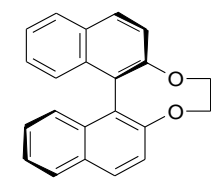

(R)-PL2
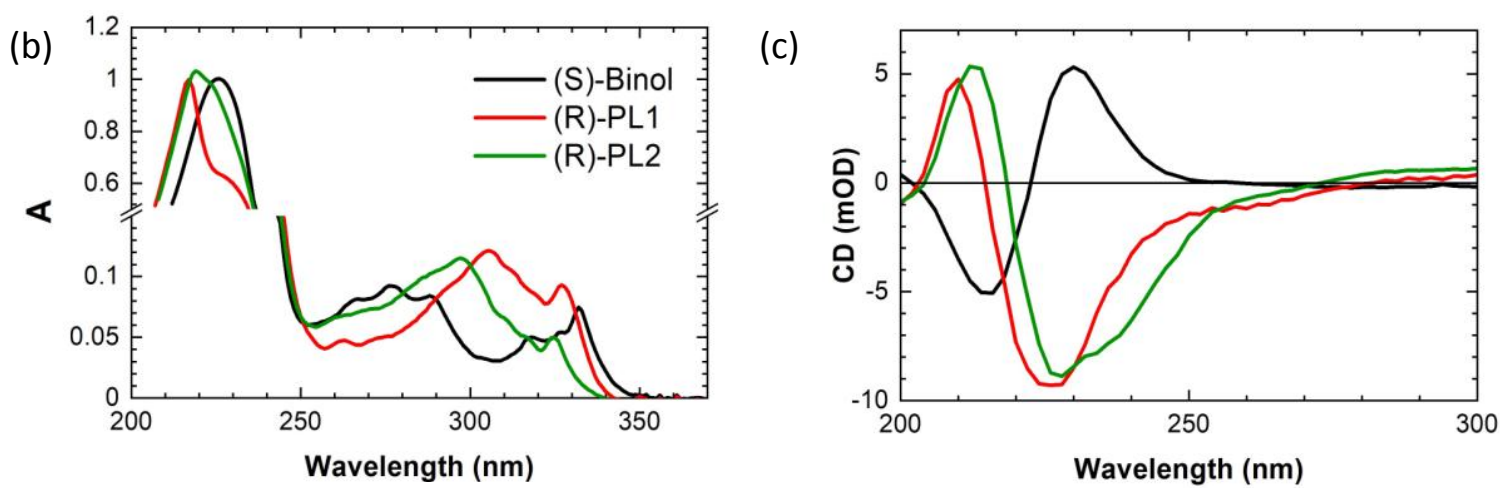

Figure 1: (a) Schematic representation of the R-binol atropoisomer and its two derivatives, (R)-PL1 and (R)-PL2. (b) Absorption and (c) CD spectra of the three compounds in cyclohexane and ethanol, respectively.

We have investigated the conformational dynamics of the binol and two derivatives depicted in Fig. 1a, after UV excitation. Fig. 1b and 1c represent their absorption and CD spectra. The two derivative exhibit the characteristic bisignate CD spectrum of the binol. All those features could be well reproduced by TD-DFT calculations [30]. Calculations also revealed a correlation between the amplitude of the $\mathrm{CD}$ bisignate and the existence of a large distribution of the dihedral angles in the binol. This distribution is strongly reduced in PL1 and PL2 due to the presence of the short bridges between the two naphthol moieties leading to the increase of the CD signals around $230 \mathrm{~nm}$.

The TRCD of the binol and its two derivatives has been probed at $234 \mathrm{~nm}$ with the ellipticity technique using a Babinet-Soleil compensator, after excitation at $266 \mathrm{~nm}$ with 100fs laser pulses. Fig. 2 represents typical TRCD signals measured for the binol and PL1 dissolved in ethanol, cyclohexane or ethylene glycol. These signals reflect the dynamical changes in the dihedral angle after the photoexcitation. They are strongly solvent-dependent. Relaxation in the viscous ethylene glycol is much slower that in the other less viscous solvents, emphasizing the fact that the observed CD changes are indeed due to the rotational motion of the naphthol moieties which is slowed down by the solvent viscosity. On the other 
hand, the unbridged binol exhibits significantly slower dynamics in ethanol than in cyclohexane. This difference was attributed to the presence of hydrogen bonds between the O-H groups of binol and ethanol. On the contrary, PL1 and PL2 which cannot form such hydrogen bonds show similar dynamics in cyclohexane and ethanol.
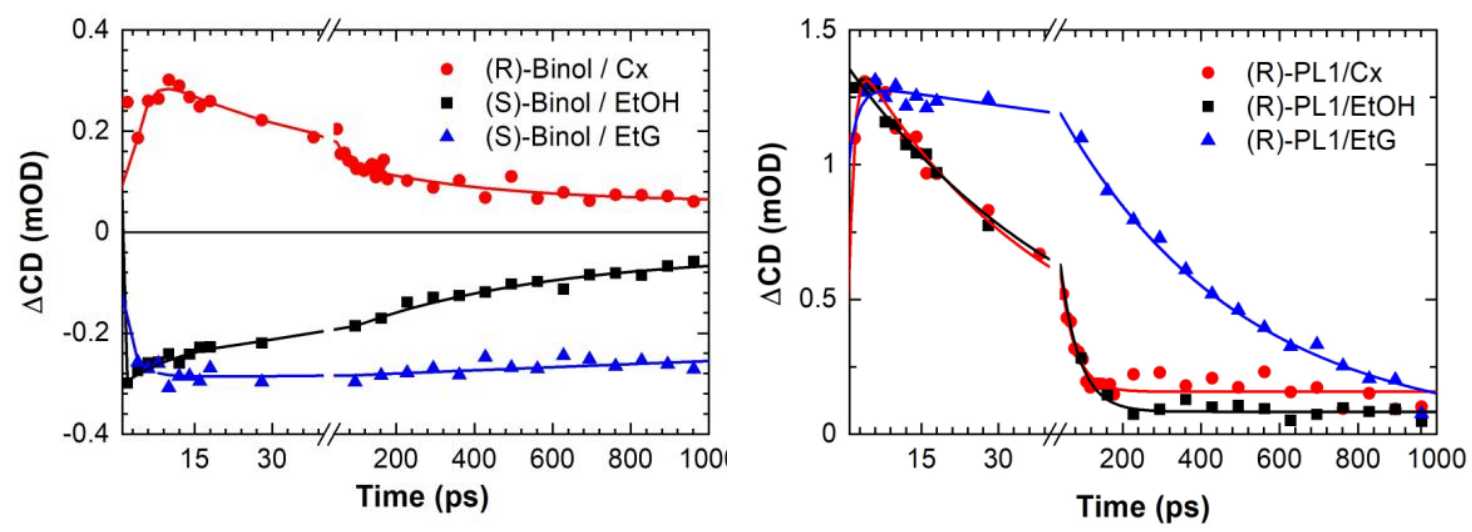

Figure 2: (a) Schematic representation of the R-binol atropoisomer and its two derivatives, $(R)$-PL1 and (R)-PL2. (b) Absorption and (c) CD spectra of the three compounds in cyclohexane and ethanol, respectively.

Quantum calculations have also revealed that these dynamics are in fact a subtle interplay between the electronic and the structural changes of the compounds upon excitation. Additional fluorescence measurements provided evidence of the inversion of the two lowest $\pi \pi *$ states $\left({ }^{1} \mathrm{~L}_{\mathrm{a}}\right.$ and ${ }^{1} \mathrm{~L}_{\mathrm{b}}$ states in Platt's notation) after excitation, for PL1. [30]

\section{2) Photodissociation of carboxy-myoglobin}

Myoglobin is a 153-residue protein involved in the storage of oxygen in muscles. Ligands such as $\mathrm{O}_{2}$ or $\mathrm{CO}$ can be fixed in the heart of the protein on a heme. Many studies have been devoted to understanding of the structural changes of the protein following the ligand dissociation. Ultrafast laser pulses have been used since the 1980's to induce the CO detachment from the heme to follow the conformational changes of the protein induced by the ligand departure. In the years 2000 [31], we have developed a TRCD experiment to bring a new light on this process, relying on the difference in the steady-state CD spectra of the carboxy (MbCO) and the deoxy (Mb) forms of myoglobin (Fig. 3).
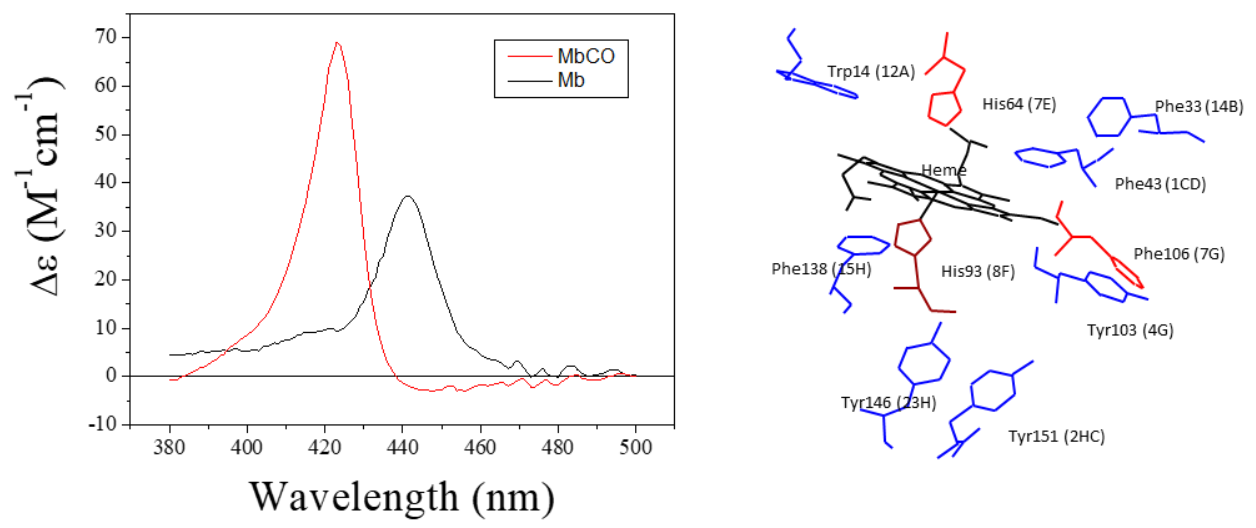
Figure 3: $C D$ spectra of carboxy(MbCO)- and deoxy(Mb)-myoglobin in the Soret band Sketch of the aromatic amino acids surrounding the heme, responsible for the CD signal [32].

TRCD was carried out with a sub-picosecond laser delivering pulses tunable in the visible spectral domain. The photodetachment of the CO ligand was achieved with 400-nm laser pulses. The dynamics of the induced $\mathrm{CD}$ changes were monitored with laser pulses tuned in the Soret band (420-440 nm) of the myoglobin. Those measurements were achieved thanks to the combination of a Pockels cell and a lock-in amplifier [31]. Fig. 4 shows the measured TRCD signals at $422 \mathrm{~nm}$ corresponding to the CD maximum for $\mathrm{MbCO}$ and at $440 \mathrm{~nm}$ corresponding to the $\mathrm{CD}$ maximum for $\mathrm{Mb}$. The figure also displays in the inset the transient absorption signals measured at the same wavelengths. Their temporal evolution are quite different from the TRCD ones, indicating that these latter are related to conformational changes of the heme environment. Note that the peaks at the zero time delay stem from an optical artefact and give the temporal resolution of the experiment. The CD changes at longer time delays exhibit a decrease at $422 \mathrm{~nm}$ in a few tens of ps concomitant to an increase at 440 $\mathrm{nm}$ which correspond to the disappearance of $\mathrm{MbCO}$ and the appearance of $\mathrm{Mb}$. Calculation of the $\mathrm{MbCO} / \mathrm{Mb} \mathrm{CD}$ spectra in the Applequist's polarizability theory, [32] considering the geometry of $\mathrm{MbCO}$ and $\mathrm{Mb}$ and the coupling of all the aromatic residues surrounding the heme (see fig.3), showed that this effects stemmed from the local deformation of the proximal histidine (His93) following the doming of the heme after the $\mathrm{CO}$ dissociation [33]. Remarkably, this local deformation which is over in a few tens of picoseconds triggers the large-scale changes in the protein conformation.

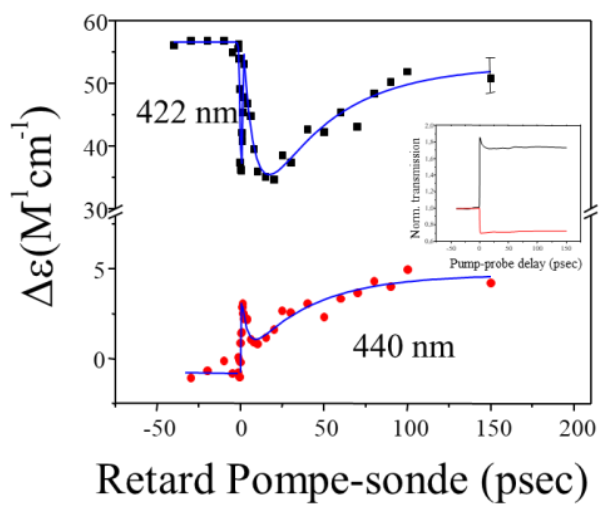

Figure 4: Circular dichroism for $\lambda=422 \mathrm{~nm}$ (close to $\mathrm{MbCO}$ peak) and $\lambda=440 \mathrm{~nm}$ (close to $M b$ peak) as a function of the pump-probe delay. The CD curves are fitted by two exponential functions (7 and 43 ps). The inset shows the corresponding normalized transmission curves.

The two experiments, we described here, are good examples of what can be typically observed with TRCD experiments in the pico-nanosecond time scale. Notably, such experiments are able to capture very rapid conformational changes in chiral systems, bringing structural information that are difficult to access with standard time-resolved achiral spectroscopy.

\section{Micro-millisecond timescale}


The previous two experiments revealed in both cases conformational changes occurring in a few hundreds of picoseconds, that is a very short timescale for processes involving the real motion of atoms and molecules. The reason why so rapid phenomena occur is because they only concern local changes and the atoms responsible for these changes only move over a few nanometer range. However, there are many interesting conformational changes that imply the global motion of a large number of atoms over much larger distance. Among them, we have been interested in folding/unfolding of biological samples, either proteins or DNA structures. These processes are of prime interest in biology because it is well known that the mechanisms underlying the biological function of those compounds are strongly dependent on their 3Dstructure. We have therefore implemented a Temperature-jump experiment where an IR laser light is used to suddenly heat up the water in which are dissolved the protein or DNA molecules that subsequently undergo an unfolding process. In that case, the samples go all the way from a densely packed arrangement of the individual components to a randomly-oriented distribution. As expected, these large scale motions do not occur on nanosecond timescales but require longer times in the microsecond range or beyond.

To address this issue and have access to long timescales in the TRCD, we implemented another set-up [34] where the probe is now a quasi-cw UV laser beam that is sent through the sample and measured by a rapid photomultiplier tube coupled to a $500 \mathrm{MHz}$ oscilloscope. Thanks to this set-up, we were able to measure directly the time evolution of the optical transmission of the sample with a microsecond resolution. To access the CD, we used the standard set-up consisting in modulating the polarization of the probe with a photoelastic modulator and deconvoluting the absorption modulation due to the $\mathrm{CD}$ with a lock-in amplifier. The final resolution of this device is then limited by the integration time constant of the lock-in amplifier.

\section{1) Thermal denaturation of a poly(Glutamic acid) peptide}

The first example we want to describe is the thermal denaturation of a short peptide made of 21 glutamic acid residues (PGA) [22]. This sample was chosen because it is known that this polypeptide assumes an $\alpha$-helical shape at low temperature and undergoes denaturation towards a random coil at higher temperature. It has therefore been intensively studied as a paradigm of helix formation in proteins. However, most of the experiments aiming to investigate the short-time dynamics of the process have relied on IR absorption of amide I' bands that have constrained the experiments to use heavy water as solvent. Thanks to TRCD, we have been able to perform the experiments in natural water as well as in heavy water. We have thus investigated the thermal unfolding of PGA dissolved in both solvents. For natural water, temperature jump is obtained with a $1.5 \mu \mathrm{m}$ pulse whereas for heavy water, it is 1.9 $\mu \mathrm{m}$. $\alpha$-helices have a characteristic CD feature around 220nm (see inset of Fig. 5a) and was used to monitor the helical fraction of the samples. As can be seen in Fig. 5a, there is a transition between the folded and the unfolded states around $330 \mathrm{~K}$, but with a much smoother transition in natural water compared to heavy water. 

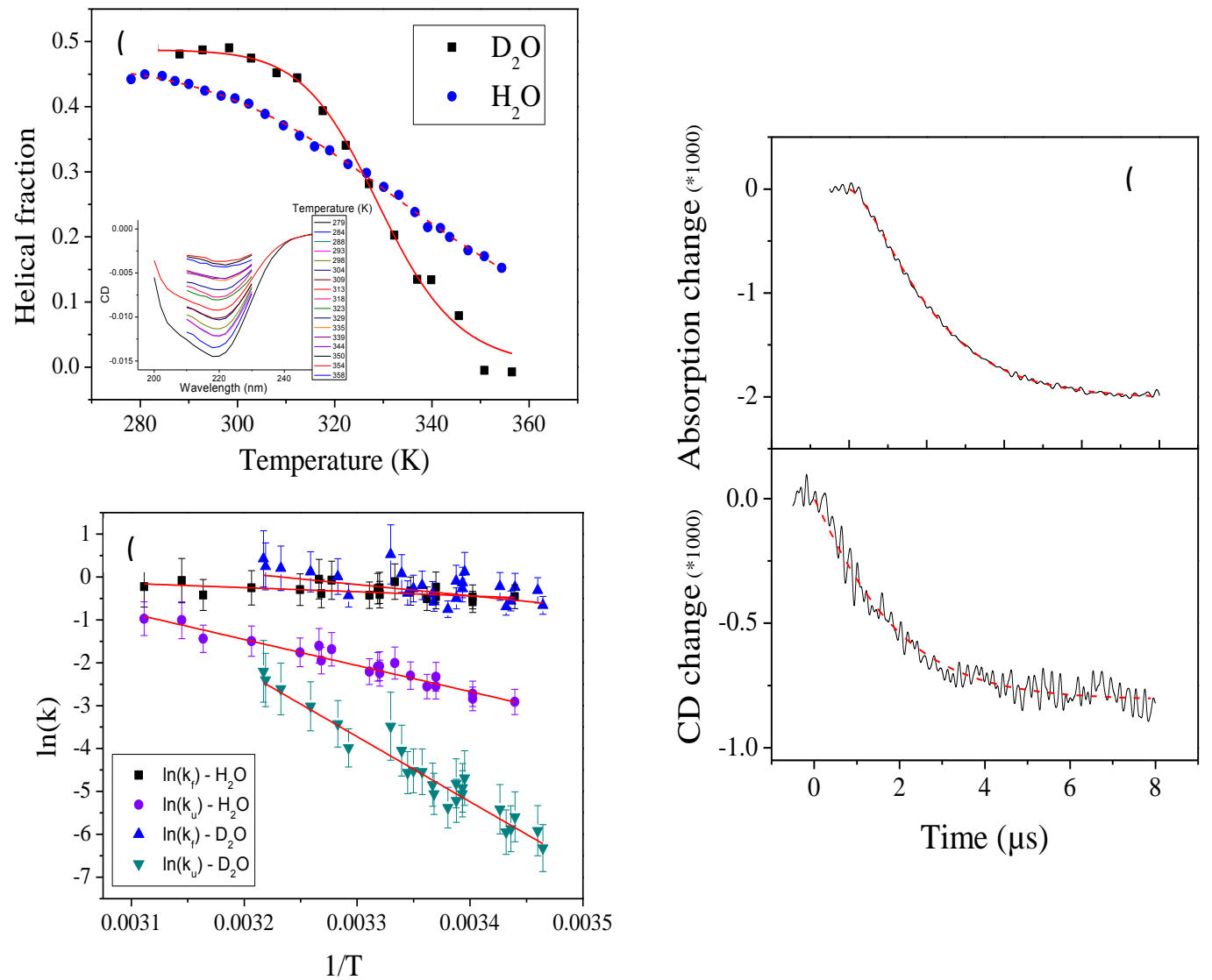

Figure 5: (a) Helical fraction for PGA dissolved in light water for $p H=4.7$ (blue dots) and in heavy water for $p D=4.7$ ( black squares) as a function of the temperature. The inset shows steady-state $\mathrm{CD}$ spectra in $\mathrm{H}_{2} \mathrm{O}$ for temperatures ranging between 279 and $358 \mathrm{~K}$. (b) Absorption change and $\mathrm{CD}$ change in $\mathrm{PGA}$ in $\mathrm{H}_{2} \mathrm{O}(\mathrm{pH}=4.7)$ for a final temperature of 299 $K$ as a function of time. The solid lines correspond to monoexponential fits with a time constant of $1.3 \mu \mathrm{s}$. (c) Arrhenius plots for the folding/unfolding reaction rates in $\mathrm{H}_{2} \mathrm{O}, \mathrm{pH}=$ 4.7 and in $\mathrm{D}_{2} \mathrm{O}, \mathrm{pD}=4.7$. The solid red lines are linear fits yielding the activation energies.

A 4-8 K temperature jump was obtained with a $5 \mathrm{~ns}$ IR pulse sent onto a $100 \mu \mathrm{m}$-thick cell and could be maintained for about $1 \mathrm{~ms}$. The probe beam was tuned in the far-UV by frequency-quadrupling of a femtosecond titanium-sapphire laser whose circular polarization was modulated with a Pockels cell at $120 \mathrm{~Hz}$. A typical TRCD curve is depicted in Fig. $5 \mathrm{~b}$ and one can measure a single exponential relaxation with a $1.3 \mu$ s time response that corresponds to the unfolding time for a temperature of $299 \mathrm{~K}$. Experiments have been carried out for different starting temperatures and for both solvents. The folding/unfolding reaction of PGA is well described by a two-state equilibrium, and the measured relaxation rate is actually the sum of the folding and unfolding reaction rates; however, the joint knowledge of the melting curves (Fig 5a) and the TRCD (Fig. 5b) can yield the individual rates (see ref. [22] for details) and it has been possible to draw the Arrhenius plots independently for the folding and the unfolding processes. As can be seen in Fig. 5c, one obtains straight lines that yield the activation energies for both processes, for natural water or heavy water. The results are summarized in Table 1It is noteworthy that the values are quite different for natural and heavy 
water, the activation energies being notably larger in the latter case. Several features could explain this feature, such as the difference in the hydrogen bond strength or in the $\mathrm{pK}$ values in the two solvents. As seen in Table 1, the experiments in natural water have been performed for several $\mathrm{pH}$ values and activation energies were shown to decrease when $\mathrm{pH}$ increases. This feature is related to the fact that at lower $\mathrm{pH}$, the glutamate side chains are more charged which decreases the helical stability.

\begin{tabular}{|c|c|c|}
\hline $\mathbf{p H} / \mathbf{p D}$ & Folding activation energy & Unfolding activation energy \\
\hline $4.2\left(\mathrm{H}_{2} \mathrm{O}\right)$ & $21 \pm 4$ & $66 \pm 5$ \\
\hline $4.4\left(\mathrm{H}_{2} \mathrm{O}\right)$ & $11 \pm 2$ & $56 \pm 3$ \\
\hline $4.7\left(\mathrm{H}_{2} \mathrm{O}\right)$ & $7 \pm 3$ & $50 \pm 4$ \\
\hline $5.0\left(\mathrm{H}_{2} \mathrm{O}\right)$ & $6 \pm 5$ & $48 \pm 6$ \\
\hline $4.7\left(\mathrm{D}_{2} \mathrm{O}\right)$ & $21 \pm 6$ & $128 \pm 6$ \\
\hline
\end{tabular}

Table 1: Folding and unfolding activation energies (in $\mathrm{kJ} / \mathrm{mol}$ ) for PGA in various solvents and $\mathrm{pH}$ conditions.

\section{2) Unfolding/refolding of DNA G-quadruplex}

G-quadruplexes (G4) are non-canonic structures of DNA that can be formed in guanine-rich sequences. In those structures, four guanine bases assemble to form a quartet stabilized by a cation $\left(\mathrm{K}^{+}\right.$or $\left.\mathrm{Na}^{+}\right)$and two or three quartets then pile up to form the quadruplex. The $\mathrm{G} 4$ can assume various configurations that can be parallel, antiparallel or hybrid depending on the orientation of the various guanine strands. These structures are known to exist in cells under physiological conditions and are suspected to play a regulation role in the gene expression. They could also be present in the chromosome telomeres and be potential target for anticancerous drugs. As such, study of G-quadruplexes has been very active lately [35]. However, very little is known about the thermodynamic and kinetic characteristics of their formation. Actually, CD spectra reveal characteristic features that depend on the specific configuration of the G4s and TRCD can therefore bring new information on these processes. We have thus implemented a T-jump experiment coupled to TRCD detection for that purpose. The main issue here is that the characteristic times involved in these processes are in the hundred of milliseconds range, much longer than the previously accessible times. We have therefore come up with another T-jump set-up where a cw IR diode is used to continuously heat up the samples. Switching on and off the IR beam is controlled by rapid shutters, allowing heating of the samples within a few tens of ms [5]. The experimental procedure, as observable in Fig. 6a, is as follows: During the first second, there is no illumination, allowing the measurement of the steady-state CD, then IR light is switched on for 4 seconds, provoking a rapid heating of the sample and the subsequent denaturation of the G4. This effect translates into a decrease of the $\mathrm{CD}$ as observable in Fig. 6a. The light is then switched off for 6 seconds, the temperature rapidly goes back to the initial value forcing the $\mathrm{G} 4$ to renaturate and the $\mathrm{CD}$ comes back to its original value. This experiment is performed for various initial temperatures and the denaturation and renaturation times of the G4 are extracted from the experimental curves, taking into account the real dynamics of the heating/cooling process. 

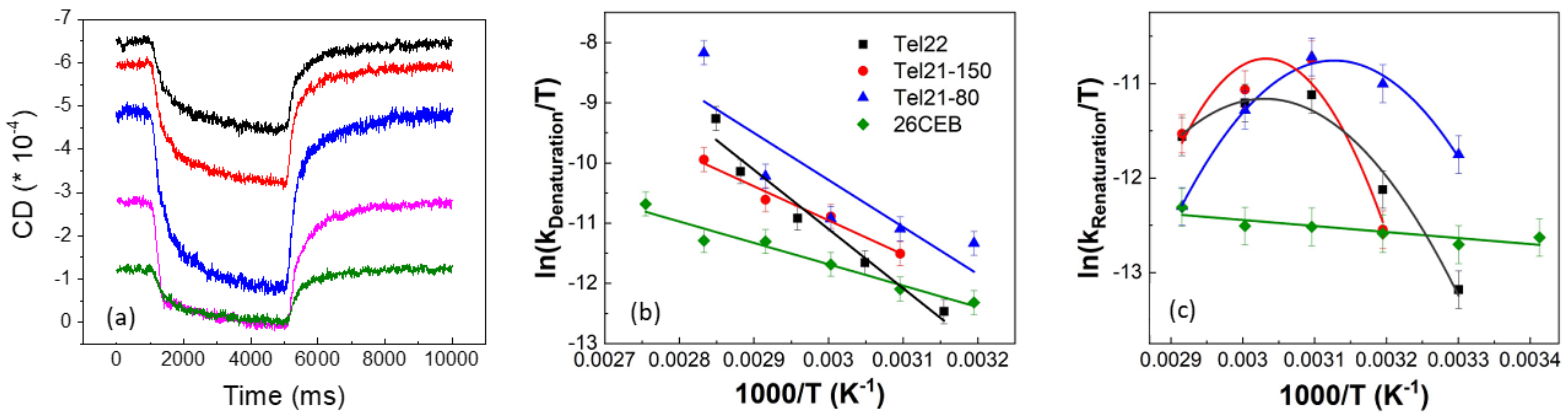

Figure 6: (a) CD changes measured at $293 \mathrm{~nm}$ for Tel21 with $80 \mathrm{mM} \mathrm{Na}^{+}$, (b-c)Variation as function of the temperature of $(b)$ the denaturation and $(c)$ the renaturation rates of Tel 1 (150 and $80 \mathrm{mM} \mathrm{Na}{ }^{+}$), Tel22 and 26CEB extracted from time-resolved CD measurements. Solid lines illustrate the linear and the parabolic fits of the experimental data.

We have studied four different samples. Three of them (two Tel21 and one Tel22) are telomeric samples, which assume an antiparallel structure whereas the fourth one (CEB26) is a parallel G4. As can be seen in Fig. 7, all samples have a transition between a folded state and an unfolded one that one can monitor through the CD peaks in the redder part of the CD spectra.
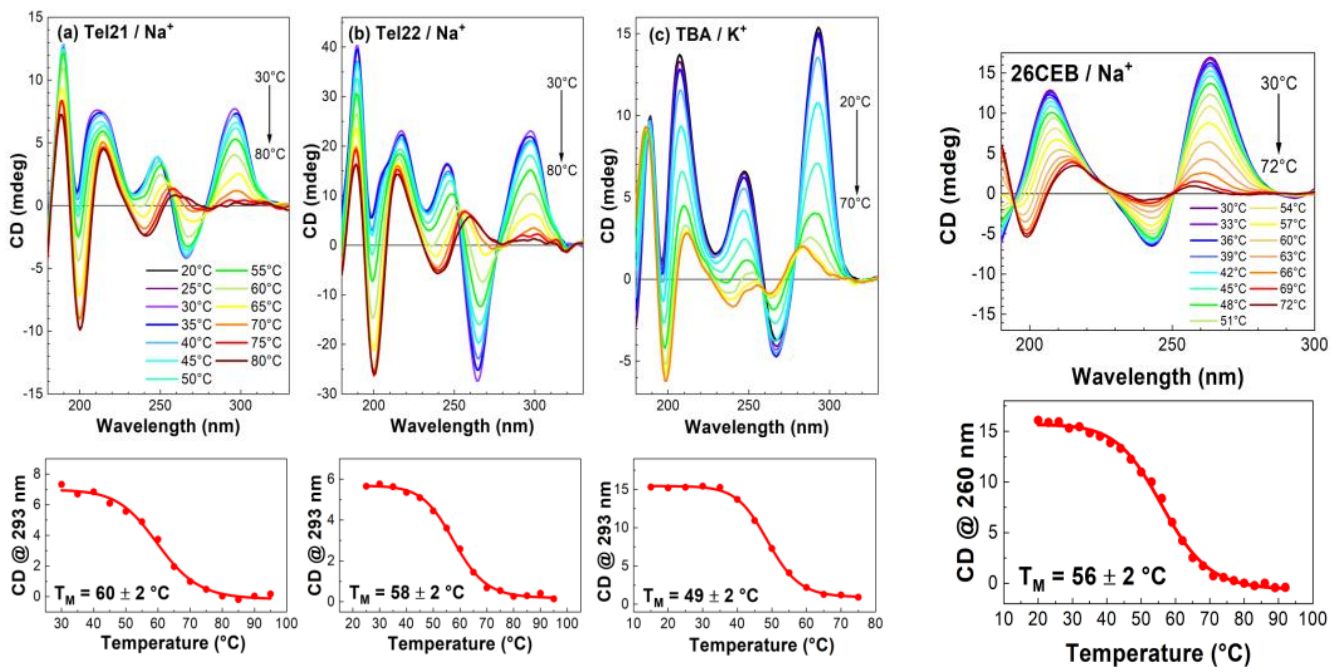

Figure 7: Synchrotron radiation CD spectra and extracted melting curves at $293 \mathrm{~nm}$ for (a) Tel21, (b) Tel22 with $150 \mathrm{mM} \mathrm{Na}^{+}$, (c) TBA with $150 \mathrm{mM} \mathrm{K} \mathrm{K}^{+}$and (d) CEB26, measured for increasing temperatures with a step of $5^{\circ} \mathrm{C}$.

We have therefore been able to measure the characteristic times for the denaturation as well as the renaturation processes. The corresponding transition rates are plotted as Eyring plots in Fig. 6b,c. The first striking feature is that denaturation end renaturation processes are clearly two very different phenomena, ruling out a simple two-state description. Actually, denaturation is more rapid that renaturation, revealing a complex energy landscape full of 
traps that prevent the rapid refolding of unfolded G4 in agreement with Ref. [36]. Considering first the denaturation processes, one can see that the curves are compatible with an activation energy in the $10 \mathrm{kcal} / \mathrm{mol}$ range. Interestingly, the CEB26 energy is lower than the telomeric ones, as expected from the lesser stability of the former. On the other hand, one can see that renaturation processes are strikingly different for the telomeric $\mathrm{G} 4 \mathrm{~s}$ that display bell-shaped Eyring curves, reminiscent of what has been observed in protein folding [37]. Interpretation of this feature is similar : at low temperature, the relaxation is driven by a weak activation energy, whereas at higher temperature, the process is entropy-driven, limited by the very large energy landscape that must be probed by unfolded G4s. Interestingly, such is not the case for the parallel G4 that displays a regular Eyring plot also for renaturation. These experiments emphasize the importance of the loops that exist between the guanine quartets in the G4 that determine the complexity of the energy landscape and thus the dynamics of renaturation of those systems.

\section{Conclusion}

TRCD remains a technique of choice for investigating the dynamics of conformational changes in (bio)molecules. Thanks to the multiplication of techniques, it is now possible to cover a very large temporal domain, form pico-nanosecond times up to seconds and to observe many different phenomena, involving either very local motions or large-scale global effects. The experiments presented here reflect the activity developed in our group, but many recent technological achievements aiming to implement broadband measurements with an improved sensitivity have been obtained recently and there is no doubt that this research field will notably expand and bring invaluable information in the next future. 


\section{References}

1. Berova N, Nakanishi K, Woody R W, Circular dichroism - Principles and applications - 2nd ed. 2000, New York: Wiley-VCH.

2. Mesnil H, Hache F, Experimental evidence of third-order nonlinear circular dichroism in a liquid of chiral molecules. Phys. Rev. Lett., 2000; 85: p. 4257-4260.

3. Björling S C, Goldbeck R A, Milder S J, Randall C E, Lewis J W, Kliger D S, Analysis of Optical Artifacts In Ellipsometric Measurements of Time-Resolved Circular Dichroism. J. Phys. Chem., 1991; 95(4685).

4. Hache F, Invited featured article : Application of time-resolved circular dichroism to the study of conformational changes in photochemical and photobiological processes. J. Photochem. Photobiol. A, 2009; 204: p. 137-143.

5. Changenet $\mathrm{P}$, Hache F, T-jump and circular dichroism: folding dynamics in proteins and DNA. Mol. Crsyt. Liq. cryst., 2019; 693: p. 49-56.

6. Lewis J W, Tilton R F, Einterz C M, Milder S J, Kuntz I D, Kliger D S, New technique to measure circular dichroism changes on a nanosecond timescale. Application to (carbonmonoxy)myoglobin and (carbonmonoxy)hemoglobin. J. Phys. Chem., 1985; 89: p. 289-294.

7. Xie X, Simon J D, Picosecond time-resolved circular dichroism spectroscopy: experimental details and applications. Rev. Sci. Instrum., 1989; 60: p. 2614-2626.

8. Dartigalongue T, Hache F, Precise alignment of a longitudinal Pockels cell for timeresolved circular dichroism experiments. J. Opt. Soc. Am. B, 2003; 20: p. 1780-1787.

9. Niezborala $\mathrm{C}$, Hache F, Measuring the dynamics of circular dichroism in a pumpprobe experiment with a Babinet-Soleil compensator. J. Opt. Soc. Am. B, 2006; 23: p. 2418-2424.

10. Trifonov A, Buchvarov I, Lohr A, Würthner, Fiebig T, Broadband femtosecond circular dichroism spectrometer with white-light polarization control. Rev. Sci. Instr., 2010; 81: p. 043104.

11. Mangot L, Taupier G, Romeo M, Boeglin A, Cregut O, Dorkenoo K D H, Broadband transient circular dichroism spectroscopy in chiral molecules. Opt. Lett., 2010; 35: p. 381-383.

12. Hiramatsu K, Nagata $\mathrm{T}$, Brodband and ultrasensitive femtosecond time-resolved circular dichroism spectroscopy. J. Chem. Phys., 2015; 143: p. 121102.

13. Oppermann M, Bauer B, Rossi T, Zinna F, Helbing J, Lacour J, Chergui M, Ultrafast broadband circular dichroism in the deep utraviolet. Optica, 2019; 6: p. 56-60.

14. Sanda F, Mukamel S, Novel coherent two-dimensional optical spectroscopy probes of chirality exchange and fluctuations in molecules. J. Chem. Phys., 2011; 135: p. 194201.

15. Holdaway D I H, Collini E, Olaya-Castro A, Isolating the chiral contribution in optical two-dimensional chiral spectroscopy using linearly polarized light. Opt. Expr., 2017; 25: p. 6383-6401.

16. Fidler A F, Singh V P, Long P D, Dahlberg P D, Engel G S, Dynamic localization of electronic excitation in photosynthetic complexes revealed with chiral twodimensional spectroscopy. Nat. Comm., 2014; 5: p. 3286.

17. Helbing J, Bonmarin M, Time-resolved chiral vibrational spectroscopy. Chimia Int. J. Chem., 2009; 63: p. 128-133.

18. Rhee H, June Y G, Lee J S, Lee K K, Ha J H, Kim Z H, Jeon S J, Cho M J, Femtosecond characterization of vibrational optical activity of molecules. Nature, 2009; 458: p. 310-313. 
19. Bonmarin M, Helbing J, A picosecond time-resolved vibrational circular dichroism spectrometer. Opt. Lett., 2008; 33: p. 2086.

20. Kliger D S, Lewis J W, Fast time-resolved circular dichroism measurements, in Circular dichroism - Principles and applications, N. Berova, K. Nakanishi, and R.W. Woody, Editors. 2000; Wiley-VCH: New-york. p. 243-259.

21. Lewis J W, Goldbeck R A, Kliger D S, Xie X, Dunn R C, Simon J D, Time-Resolved Circular Dichroism Spectroscopy: Experiment, Theory, and Applications to Biological Systems. J. Phys. Chem., 1992; 96: p. 5243-5254.

22. Mendonça L, Steinbacher A, Bouganne R, Hache F, Comparative study of the folding/unfolding dynamics of poly(glutamic acid) in light and heavy water. J. Phys. Chem. B, 2014; 118: p. 5350-5356.

23. Meyer-Ilse J, Akimov D, Dietzek B, Ultrafast circular dichroism study of the ring opening of 7-dehydrocholesterol. J. Phys. Chem. Lett., 2012; 3: p. 182-185.

24. Scholtz M, Morgenroth M, Cho M J, Choi D H, Oum K, Lenzer T, Ultrafast broadband transient absorption and circular dichroism reveal relaxation of a chiral coploymer. J. Phys. Chem. Lett., 2019; 10: p. 5160-5166.

25. Oppermann M, Spekowius J, Bauer B, Pfister R, Chergui M, Helbing J, Broad-band ultraviolet CD spectroscopy of ultrafast peptide backbone conformational dynamics. $J$. Phys. Chem. Lett., 2019; 10: p. 2700-2705.

26. Hache F, Time-resolved circular dichroism : what can we learn on conformational changes. Display and Imaging, 2015; 3-4: p. 197-209.

27. Schmid M, Martinez-Fernandez L, Markovitsi D, Santoro F, Hache F, Improta R, Changenet P, Unveiling Excited-State Chirality of Binaphthols by Femtosecond Circular Dichroism and Quantum Chemical Calculations. J. Phys. Chem. Lett., 2019; 10: p. 4089-4094.

28. Kuronuma M, Sato T, Araki Y, Mori T, Sakamoto S, Inoue Y, Ito O, Wada T, Transient circular dichroism of the excited triplet state of pristine hexahelicene in solution at room temperature. Chem. Lett., 2019; 48: p. 357-360.

29. Auvray F, Dennetiere D, Giulani A, Jamme F, Wien F, Nay B, Zirah S, Polack F, Menneglier C, Lagarde B, Hirst J D, Réfrégiers M, Time resolved transient circular dichroism spectroscopy using synchrotron natural polarisation. Structural Dynamics, 2019; 6: p. 054307.

30. Hache F, Unveiling excited-state chirality of binaphthols by sub-picosecond circular dichroism and quantum-chemical calculations, in Photonics West 2020. 2020: San Francisco.

31. Dartigalongue $\mathrm{T}$, Hache $\mathrm{F}$, Observation of sub-100 ps conformational changes in photolyzed carbonmonoxy-myoglobin probed by time-resolved circular dichroism. Chem. Phys. Lett., 2005; 415: p. 313-316.

32. Dartigalongue T, Hache F, Calculation of the circular dichroism spectra of carbon monoxy-and deoxy-myoglobin: Interpretation of a time-resolved circular dichroism experiment. J. Chem. Phys., 2005; 123: p. 184901-1/9.

33. Dartigalongue $\mathrm{T}$, Hache $\mathrm{F}$, Time-resolved circular dichroism in carbonmonoxymyoglobin: the central role of the proximal histidine. Chirality, 2006; 18: p. 273-278.

34. Khuc M T, Mendonça L, Sharma S, Solinas X, Volk M, Hache F, Measurement of circular dichroism dynamics in a nanosecond temperature-jump experiment. Rev. Sci. Instrum., 2011; 82: p. 054302 (8p).

35. Spiegel J, Adhikari S, Balasubramanian S, The structure and function of DNA Gquadruplexes. Trends in Chemistry, 2019.

36. Sponer J, Bussi G, Stadbauer P, Kührova P, Banas P, Islam B, Haider S, Neidle S, Otyepka M, Folding of guanine qudruplex molecules-funnel-like mechanism or 
kinetic partitioning? An overview from MD simulation studies. Biochimica et Biophysica Acta, 2017; 1861: p. 1246-1263.

37. Dobson C M, Sali A, Karplus M, Protein folding: A perspective form theory and experiment. Angew. Chem. Int. Ed., 1998; 37: p. 868-893. 